\title{
Radiation Effect Mechanisms in Electronic Devices
}

\author{
M. A. G. da Silveira', R. B. B. Santos, F. Leite, F. Cunha, K. H. Cirne \\ Centro Universitário da FEI \\ São Bernardo do Campo, S.P., Brazil \\ marcileiafei.edu.br
}

\section{N. H. Medina, N. Added, V. A. P. Aguiar}

Instituto de Física da USP

São Paulo, S.P., Brazil

In this work, P- and N-MOSFETs (Metal Oxide Semiconductor Field Effect Transistor) were submitted to X-ray and ion beams. CD 4007, a commercial off-the-shelf integrated circuit composed of six transistors, three P-type and three N-type, in a single package, was used. The integrated circuits were exposed to $60 \mathrm{MeV}{ }^{35} \mathrm{Cl}$ ion beams using the São Paulo 8UD Pelletron Accelerator and $10 \mathrm{keV}$ X-ray radiation, using a Shimadzu XRD-7000 X-ray diffractometer. The total dose effects due to ionizing radiation in MOSFET were analyzed. The results indicate $\mathrm{V}_{\text {th }}$ depends on the absorbed dose and dose rate. The deviation of $\mathrm{V}_{\mathrm{th}}$ is higher for P-MOS, while the change in slope is higher for N-MOS. TID (Total Ionizing Dose) caused by heavy ion does not seem to affect mobility. After heat treatment, the device establishes a different equilibrium state compared to that achieved at room temperature. The heat treatment worsens the P-type characteristics and improves the N-type.

$X$ Latin American Symposium on Nuclear Physics and Applications (X LASNPA)

1-6 December 2013

Montevideo, Uruguay

${ }^{1}$ Speaker 


\section{Introduction}

Radiation-hard and radiation-tolerant electronic devices and circuits are essential for aerospace applications. Hence, aerospace agencies and governments have an incentive to encourage scientific community to develop new techniques, fabrication processes, new materials and devices that can mitigate the degradation of the devices submitted to ionizing radiation such as protons and heavy ions, and X-and gamma-rays [1]. Radiation affects electronic components in a variety of ways, of which we single out, for this paper, parametric, and functional failures caused by charge accumulation in the gate or in the field oxides. These radiation-induced oxide-trapped charges shift the voltage threshold and the subthreshold swing, and increase the leakage current between source and drain of a transistor and also between transistors. Reliability issues and effects of ionizing radiation on electronic devices are more critical in environments where the devices are exposed to radiation, as is the case of space environments, particle accelerators, nuclear medicine and nuclear reactors facilities among others, being of strategic value for areas such as space and defense [1-4].

When electronic devices are exposed to ionizing radiation, there is a variation in the semiconductor device parameters and electrical failures. The effects caused by ionizing radiation can be transient or permanent. Transistor miniaturization, new layouts, materials and fabrication techniques caused some of these effects to be mitigated while others were enhanced. Transient effects may provoke momentary failure, for example, changes on stored data or even peaks of electrical current that might damage the electrical circuit. These are the motivations of the growing interest in this research area, since energy absorption, carrier generation, recombination, and transport, charge trapping, and defect formation influence the effects provoked by ionizing radiation [3,4]. Total ionizing dose (TID) effects are related to the amount of energy (dose) absorbed by the material. Particles with low linear energy transfer (LET), i.e. low electronic mass stopping power, and electromagnetic radiation, tend to contribute more to these effects. When photons interact with the device oxides, they create electron-hole pairs in the material, mainly by photoelectric effect. In a typical oxide, electrons can escape swiftly to the positive electrode while holes move slowly towards the negative electrode by a hopping mechanism. The slow motion of the holes increases the probability that they are trapped by defects in the bulk of the oxide or near the oxide-semiconductor interface. This positive charge concentration may change the basic operating characteristics of the device [4]. Holes can, however, escape from the oxide by tunneling or in a thermally assisted way. Thermal annealing is a technique that involves the heating of the device to a certain temperature during a certain amount of time; the thermal energy afforded to the holes stimulates their release from the traps, which may result in a recovery of initial electrical condition of the device.

In order to achieve a better understanding of the mechanisms responsible for radiation damage due to TID effects, we are studying a commercial off-the-shelf integrated circuit, the CD 4007 (manufactured by Texas Instruments). These ICs consist of three complementary pairs of N- and P-type MOSFETs, which allows us to assess the behavior of each device under test (DUT), as can be seen in Figure 1. The parameters affected by TID that are addressed in this work are the voltage threshold and the subthreshold swing shifts. Besides, a thermal annealing 
study was carried out in order to investigate the behavior of the trapped charges when activated by temperature. The DUTs were exposed to $10-\mathrm{keV} \mathrm{X}$-ray and to $60-\mathrm{MeV}{ }^{35} \mathrm{Cl}$ ion beams. The $10-\mathrm{keV} \mathrm{X}$-ray generates secondary electrons with a range of $500 \mathrm{~nm}$, comparable to the field oxide thickness.

\section{Schematic Diagram}

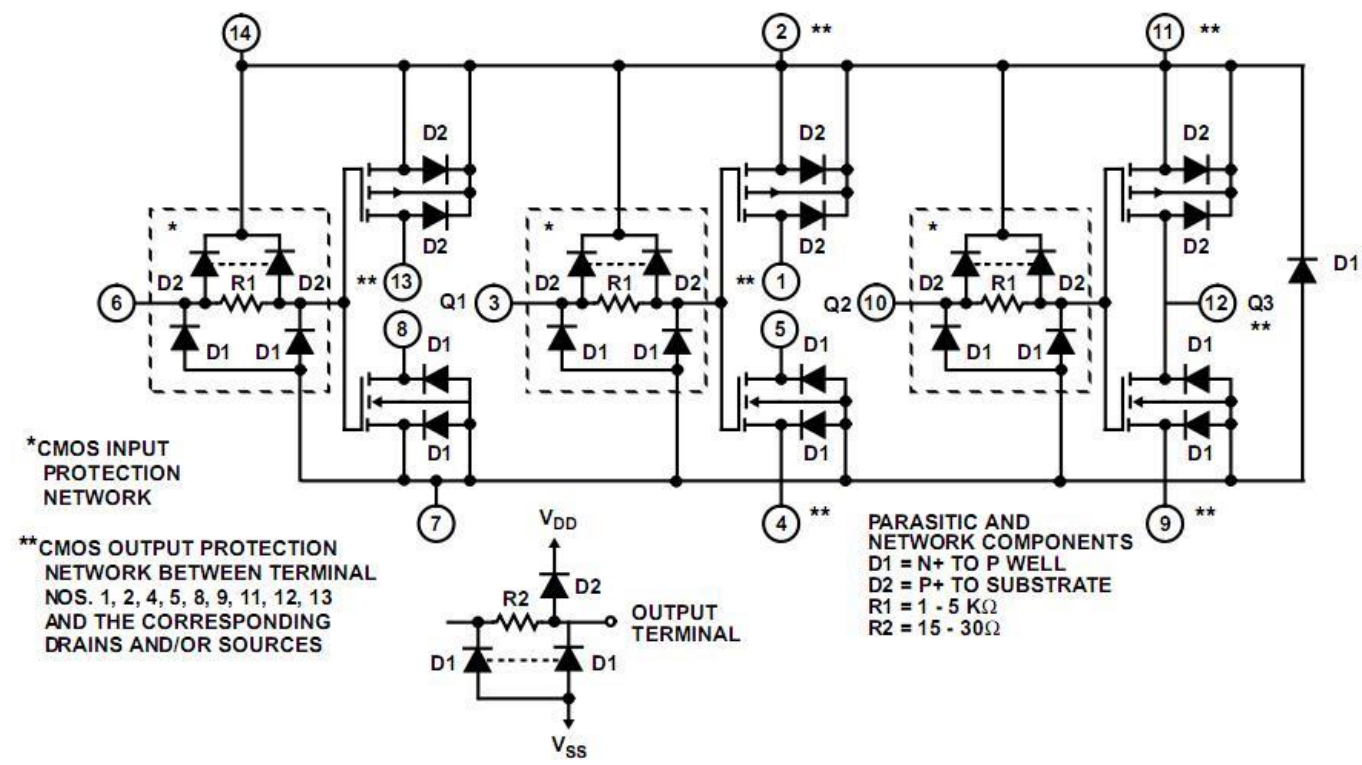

Figure 1. Detailed schematic diagram of CD4007 showing INPUT, OUTPUT and parasitic diodes. Adapted from CD4007 Texas Instrument Datasheet.

\section{Methodology}

Preparation of the devices consisted mainly in removing the epoxy layer that protects the device, reducing any interference this could have on the absorbed dose. The removal was done by etching the surface of the package with a mixture of sulphuric and nitric acids until the die were revealed. Besides, this process also allows the heavy ions to reach sensitive parts of the device. Figure 2 shows the decapsulated devices prior to irradiation.

Electrical characterizations were performed always under the same conditions using a Keithley SCS 4200 parameter analyzer. With this equipment, we obtained $I_{D} \times V_{G S}$ characteristic curves of current between source and drain $I_{D}$ as a function of gate voltage $V_{G S}$. It was determined empirically that a state of essentially permanent damage due to radiation was achieved after room-temperature annealing for about six days, i.e. the electrical characteristics of the devices settled to a steady state six days after each round of irradiation. Therefore, all electrical characterizations were performed with the devices in this steady state. 


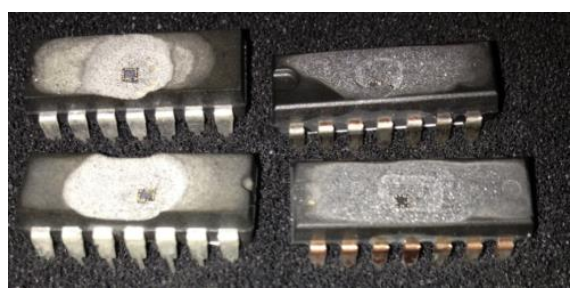

Figure 2. Decapsulated CD4007 ready to be exposed to radiation.

For the extraction of the N-MOS threshold voltage, a voltage of $100 \mathrm{mV}$ between drain and source was applied. We varied the gate voltage from $-0.5 \mathrm{~V}$ to $4 \mathrm{~V}$ in steps of $15 \mathrm{mV}$. For the extraction of the P-MOS threshold voltage, the voltage between drain and source was -100 $\mathrm{mV}$. We varied the voltage between gate and source of -0.1 to $-5.1 \mathrm{~V}$. The method for determining the threshold voltage was the second derivative of $I_{D} \times V_{G S}$ curve $[5,6]$.

\subsection{Ray irradiation}

The DUTs that are contained in IC CD4007 were exposed unbiased to a $10-\mathrm{keV}$ effective energy X ray beam in a Shimadzu XRD-7000 X-ray diffractometer. In this irradiation process, two different dose rate were used, $46 \mathrm{rad} / \mathrm{s}$, and $96 \mathrm{rad} / \mathrm{s}$. The irradiation period was controlled in order that the total doses absorbed by the devices were $100 \mathrm{krad}, 150 \mathrm{krad}, 250 \mathrm{krad}$, and 500 $\mathrm{krad}$ with both dose rates. The samples were held $10 \mathrm{~cm}$ away from the beam source to ensure homogeneity in the area to be irradiated. The dose rate was estimated by measuring exposure by an ionization chamber.

Electrical characterization of the irradiated devices were made only after they reached a steady state of radiation induced damage, as described earlier. Figure 3 shows the behavior of the threshold voltage shift as a function of time after irradiation for a N-type MOSFET, for two different dose rates with total dose of $26 \mathrm{krad}(\mathrm{Si})$. Immediately after irradiation, there is a significant shift in the threshold voltage, but the shift tends to decrease over the days reaching a steady state after a week, approximately. The P-type MOSFETs showed the same behavior for the two dose rates used in this study. The results obtained with the irradiation of $26 \mathrm{krad}$ were only used to define the parameters of tests to study the devices. 


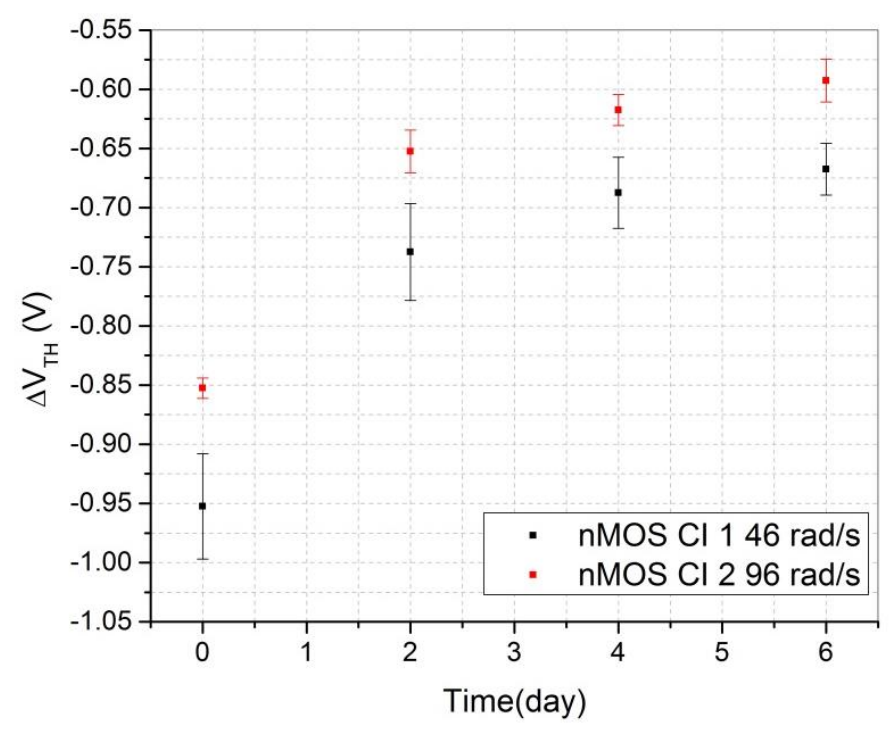

Figure 3. Change of threshold voltage fluctuation as a function of time after irradiation for N-MOSFET devices relative to the threshol before irradiation. The 0 time is the transistor before being exposed to irradiation of $26 \mathrm{krad}(\mathrm{Si})$.

\section{2. $60 \mathrm{MeV}^{35} \mathrm{Cl}$ ion beams}

In order to observe the damage due to the total ionizing dose accumulated in a process of interaction of heavy ions with the device, and compare with the damage from ionizing electromagnetic radiation, the IC CD 4007 was exposed to $60 \mathrm{MeV}{ }^{35} \mathrm{Cl}$ ion beam, with $15 \mathrm{rad} / \mathrm{s}$ (Si) dose rate, accumulated up to $80 \mathrm{krad}$. With this energy, the range of the chlorine ions on $\mathrm{Si}$ is $15 \mu \mathrm{m}$. The chlorine ions were accelerated in a $8 \mathrm{MV}$ Pelletron Accelerator of the São Paulo University, Brazil [7, 8]. To ensure that the observations would be comparables with those performed with X-ray irradiation, electrical characterization was also performed only after the devices have reached a steady state of radiation induced damage.

\section{Results and Discussion}

The results obtained in the analysis for the behavior of MOSFET devices show the influence of dose rate and cumulative dose in $\mathrm{P}$ and $\mathrm{N}$ type.

Figure 4 shows the $I_{D} \times V_{G S}$ characteristic curves of the N-type and of the P-type MOSFETs submitted to X-ray radiation with increasing total ionizing dose, and submitted to heavy ions (80 krad (Si)). For the P-type MOSFET, the current decreases monotonically as the total ionizing dose increases, voltage threshold $\mathrm{V}_{\text {th }}$ becomes more and more negative but the subthreshold swing is little affected, signaling just a small change in the carriers mobilities. For the N-type MOSFET, the situation is very different: while the subthreshold swing shifts significantly, indicating a modification in the carriers mobilities, the threshold voltage presents a noticeable negative shift at small dose; then, at larger dose, the absolute value of the threshold 
voltage shift starts to diminish. This behavior is interpreted as the buildup of positive charge in the deep traps in the oxide near the $\mathrm{Si}-\mathrm{SiO}_{2}$ interface followed by the loading of the interface traps.

Figure 4 also shows characteristic curves obtained irradiating the devices with a $60 \mathrm{MeV}$ ${ }^{35} \mathrm{Cl}$ ion beam. It is possible to observe a change in $\mathrm{V}_{\text {th }}$ but no change in subthreshold swing for the N-type devices; for the P-type, only a small change in $\mathrm{V}_{\text {th }}$ is noticeable. Considering the curves for the 80 -krad total dose generated by the ion beam and the 100-krad total dose generated by X-ray, we note that the parametric changes produced by the ion beam irradiation is less marked than the parametric changes induced by X-ray irradiation. This could be an artifact arising from the slightly smaller dose accumulated using the ion beam or it could hint at a differential damage effectiveness of the irradiation processes. Table 1 shows the values of the threshold voltage obtained before and after each irradiation testing.

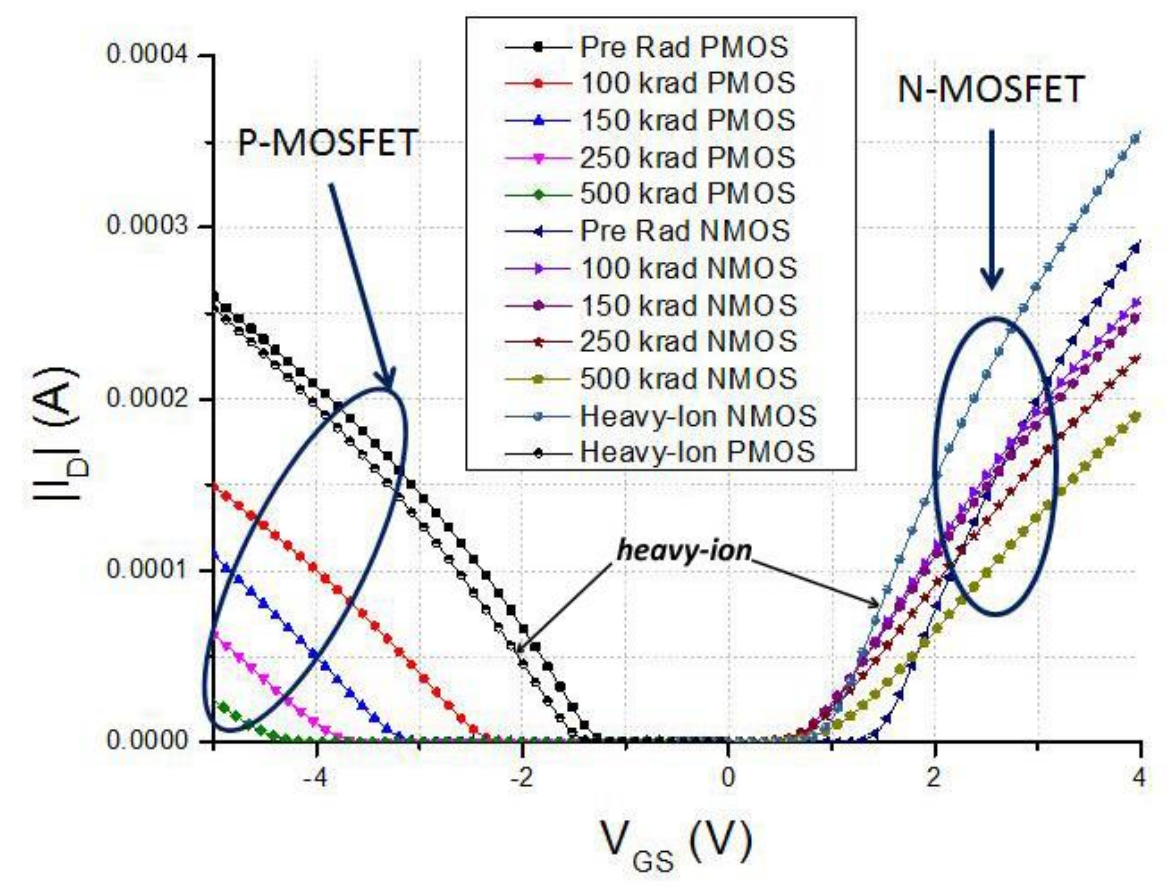

Figure 4. $\mathrm{I}_{\mathrm{D}} \mathrm{x} \mathrm{V}_{\mathrm{GS}}$ characteristic curves for different total ionizing dose in $\mathrm{P}$ and $\mathrm{N}$-MOSFET devices exposed to X-rays at a dose rate of $46 \mathrm{rad} / \mathrm{s}(\mathrm{Si})$. Also shown the results of exposure of $\mathrm{P}$ and $\mathrm{N}-\mathrm{MOSFET}$ devices to the ${ }^{35} \mathrm{Cl}$ ion beam for $80 \mathrm{krad}$ integrates dose. 
Table 1: $\mathrm{V}_{\text {th }}$ for CD4007 exposed different dose rates, different total dose and different radiation beams: X-ray and ${ }^{35} \mathrm{Cl}$ Ion beams. CI 1 is referent to $46 \mathrm{rad} / \mathrm{s}(\mathrm{Si})$ and $\mathrm{CI} 2$ is referent to $96 \mathrm{rad} / \mathrm{s}(\mathrm{Si})$.

\begin{tabular}{|c|c|c|c|c|}
\hline \multirow{4}{*}{$\begin{array}{c}\text { Total } \\
\text { Ionizing Dose }\end{array}$} & \multicolumn{4}{|c|}{ Threshold Voltage $\left(V_{\text {th }}\right)$} \\
\hline & \multicolumn{2}{|c|}{ N Type } & \multicolumn{2}{|c|}{ P Type } \\
\hline & \multicolumn{4}{|c|}{$10 \mathrm{keV}$ X-ray } \\
\hline & N-MOS CI 1 & N-MOS CI 2 & P-MOS CI 1 & P-MOS CI 2 \\
\hline Pré-Rad & $1.417 \pm 0.052$ & $1.417 \pm 0.052$ & $-1.436 \pm 0.014$ & $-1.436 \pm 0.014$ \\
\hline $100 \operatorname{krad}(\mathrm{Si})$ & $0.750 \pm 0.022$ & $0.825 \pm 0.018$ & $-2.960 \pm 0.006$ & $-2.930 \pm 0.010$ \\
\hline $150 \operatorname{krad}(\mathrm{Si})$ & $0.705 \pm 0.056$ & $0.780 \pm 0.025$ & $-3.300 \pm 0.001$ & $-3.200 \pm 0.025$ \\
\hline $250 \mathrm{krad}(\mathrm{Si})$ & $0.790 \pm 0.068$ & $0.510 \pm 0.055$ & $-3.872 \pm 0.008$ & $-3.795 \pm 0.013$ \\
\hline \multirow[t]{2}{*}{$500 \mathrm{krad}(\mathrm{Si})$} & $0.940 \pm 0.061$ & $1.035 \pm 0.082$ & $-4.420 \pm 0.030$ & $-4.315 \pm 0.210$ \\
\hline & \multicolumn{4}{|c|}{${ }^{35} \mathrm{Cl}$ Ion beam:15 rad/s } \\
\hline $80 \operatorname{krad}(\mathrm{Si})$ & \multicolumn{2}{|c|}{$0.955 \pm 0.032$} & \multicolumn{2}{|c|}{$-1.540 \pm 0.014$} \\
\hline
\end{tabular}

Figure 5 summarizes the observations made earlier regarding the voltage threshold shifts. The different behavior of the N- and P-type MOSFETs may be clearly seen. Regarding the effects of the dose rates, it may be said that there is no clear difference between the voltage threshold shifts in the P-type case for any of the total ionizing doses used.
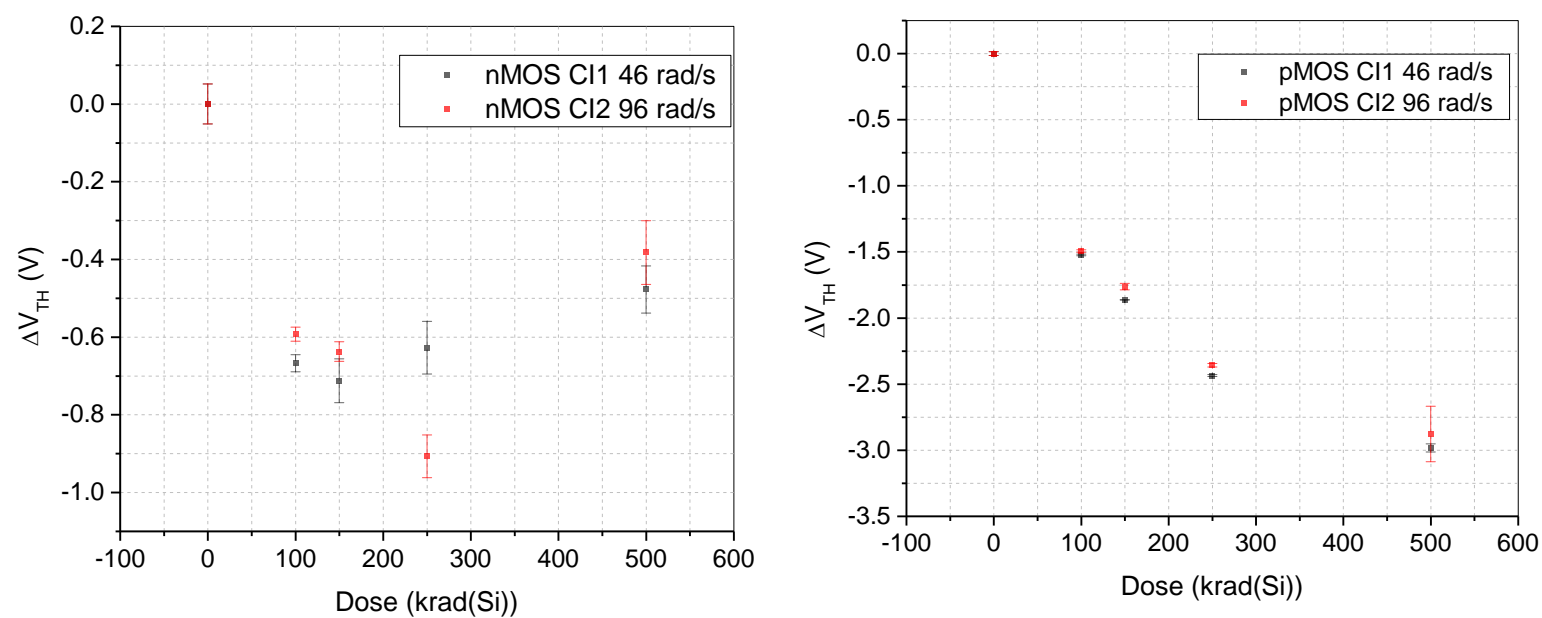

Figure 5. Variation of threshold voltage as a function of the total ionizind dose in N-MOS (left) and P-MOS devices (right).

Increasing cumulative radiation dose, the threshold voltage in P-MOS devices becomes increasingly negative, indicating that regardless of the trapped charges are predominantly in the oxide or at the interface, the current in the conduction channel always decreases. This is explained by the fact that charge carriers have the same sign of the trapped charges. It is also observed that the dose rate affects little the threshold voltage shift. On the other hand, the N-MOS devices showed a negative trend curve for the deviation in the threshold voltage of up to $150 \mathrm{krad}$ for the lower dose rate. For a dose rate of $96 \mathrm{rad} / \mathrm{s}(\mathrm{Si})$, this trend remained up to 250 krad accumulated. The responsible for the decrease in threshold voltage with the increase of the 
total dose is the trapping of positive charge in the oxide changing the electric field of the gate, increasing the conduction current in the transistor channel. With the increase of the accumulated dose for N-MOS devices, there is a rebound in behavior at the threshold voltage in $250 \mathrm{krad}$ to $46 \mathrm{rad} / \mathrm{s}(\mathrm{Si})$ and $500 \mathrm{krad}$ to $96 \mathrm{rad} / \mathrm{s}(\mathrm{Si})$. In this case, positive charges are predominantly trapped in the interface between oxide/Si, attracting negative carriers to be recombined or trapped in the same interface. These results indicate that, using the lowest dose rate, total ionizing dose provides a less number of electron-hole recombination resulting in a greater effect of radiation on the devices. This preliminary analysis shows that the dose rate is especially important for trapping mechanisms occurring in n-MOSFETs.

To investigate the behavior of the trapped charges when activated by temperature, the devices were submited to $10-\mathrm{keV} \mathrm{X}$-ray using $96 \mathrm{rad} / \mathrm{s}(\mathrm{Si})$ dose rate. The results were compared with the parameters obtained for the devices that were not submitted to a stimulated heat treatment. In a first test, after the DUT having accumulated $100 \mathrm{krad}$, they were subjected to heat treatment at $100^{\circ} \mathrm{C}$ for one hour. In a second test, the same devices were subjected to $100^{\circ}$ $\mathrm{C}$ for two hours after accumulating $250 \mathrm{krad}$, i.e., it was added a dose of $150 \mathrm{krad}$. The measurement of the drain corrent as a function of the gate voltage was performed immediately after the thermal treatment and also after one week, in each irradiation. The results, obtained after one week, are shown in Figure 6, where it is possible to observe that the temperature not only accelerates the solid state device damage, but also interfere with the mechanisms of trapping charges. It is noted that the established balance, after heat treatment, is different than the equilibrium state achieved at room temperature. The results indicate that there was a recovery of the $\mathrm{V}_{\mathrm{th}}$ value for both the n-MOS and p-MOS devices.

For a better visualization of the results obtained with the thermal annealing, Table 2 presents the value of the threshold voltage $\left(\mathrm{V}_{\text {th }}\right)$ for the $\mathrm{p}$ and n-MOSFET measured before, immediately after, and seven days after each irradiation. The $\mathrm{V}_{\text {th }}$ values measured immediately after and seven days after the thermal treatment, indicate that it is reached the stability of the damage due to TID effects after a heat treatment of $100^{\circ} \mathrm{C}$ for two hours, suggesting the temperature accelerates the process to reach stability. Moreover, comparing the results with those obtained for the same cumulative doses on devices without thermal treatment (measurements in permanent damage state, seven days after irradiation, indicated by "Room Temperature" in Table 2), we can see that the temperature has really improved the characteristics of the N-MOSFET while the characteristics of the P-MOSFET are worsened. 


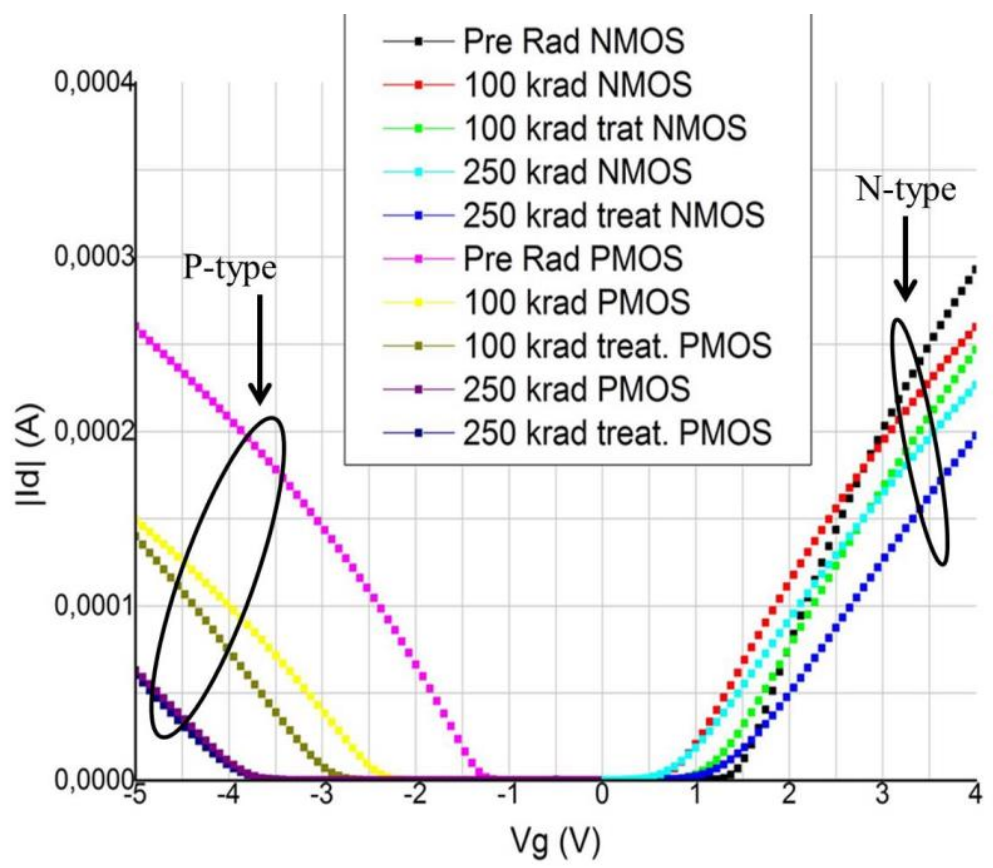

Figure 6. $\mathrm{I}_{\mathrm{D}} \times \mathrm{V}_{\mathrm{GS}}$ characteristic curves for $\mathrm{p}$ - and n-MOSFET devices, exposed to $96 \mathrm{rad} / \mathrm{s}$ dose rate with $10-\mathrm{keV} \mathrm{X}$ - rays, before and after thermal annealing.

Table 2: $\mathrm{V}_{\text {th }}$ values for $\mathrm{CD} 4007$ exposed $10-\mathrm{keV} \mathrm{X}$-ray using $96 \mathrm{rad} / \mathrm{s}(\mathrm{Si})$ dose rate. The values were obtained before and after annealing thermal at $100^{\circ} \mathrm{C}$, in DUT with $100 \mathrm{krad}$ and $250 \mathrm{krad}$ accumulated dose.

\begin{tabular}{|c|c|c|c|c|c|c|c|c|}
\hline \multirow{3}{*}{$\begin{array}{l}\text { TID } \\
(\mathrm{krad})\end{array}$} & \multicolumn{8}{|c|}{ Threshold Voltage $\left(\mathbf{V}_{\text {th }}\right)$} \\
\hline & \multicolumn{4}{|c|}{ N Type } & \multicolumn{4}{|c|}{ P Type } \\
\hline & $\begin{array}{c}\text { Room } \\
\text { Temperature } \\
7 \text { Days }\end{array}$ & $\begin{array}{c}\text { Before } \\
\text { Treatment }\end{array}$ & $\begin{array}{c}\text { After } \\
\text { Treatment }\end{array}$ & $\begin{array}{c}\text { After } \\
\text { Treatment7 } \\
\text { Days }\end{array}$ & $\begin{array}{c}\text { Room } \\
\text { Temperature7 } \\
\text { Days }\end{array}$ & $\begin{array}{c}\text { Before } \\
\text { Treatment }\end{array}$ & $\begin{array}{c}\text { After } \\
\text { Treatment }\end{array}$ & $\begin{array}{c}\text { After } \\
\text { Treatment } \\
7 \text { Days }\end{array}$ \\
\hline Pre-rad & \multicolumn{4}{|c|}{$1.480 \pm 0.053$} & \multicolumn{4}{|c|}{$-1.310 \pm 0.047$} \\
\hline 100 & $0.825(18)$ & $0.415(15)$ & $1.085(39)$ & $1.105(40)$ & $-2.930(10)$ & $-3.005(92)$ & $-3.150(93)$ & $-3.035(89)$ \\
\hline 250 & $0.510(55)$ & $1.165(42)$ & $1.325(47)$ & $1.315(47)$ & $-3.795(13)$ & $-2.980(88)$ & $-3.970(98)$ & $-3.970(98)$ \\
\hline
\end{tabular}

The results obtained after thermal annealing indicate there are holes trapped in traps that are activated with a temperature of $100^{\circ} \mathrm{C}$. Thus, the holes which were in stable equilibrium, trapped in the oxide at room temperature, are targeted by the electric field to migrate to the interface traps between oxide and $\mathrm{Si}$. This effect provokes further decrease of positive carrier current, by changing the threshold voltage of the P-MOS device. 


\section{Conclusion}

In this work, integrated circuits, $\mathrm{CD} 4007$, were exposed to $60 \mathrm{MeV}{ }^{35} \mathrm{Cl}$ ion beams using the São Paulo 8UD Pelletron Accelerator and 10-keV X-ray radiation, using a Shimadzu XRD$7000 \mathrm{X}$-ray diffractometer. Characteristic curves, in different irradiation conditions, for $\mathrm{p}$ and $\mathrm{N}$-MOSFET transistors, were studied. The results indicate $\mathrm{V}_{\mathrm{th}}$ depends on the absorbed dose and dose rate. The deviation of the $\mathrm{V}_{\text {th }}$ value is higher for P-MOS, while the change in slope is higher for N-MOS. TID (Total Ionizing Dose) caused by heavy ion does not seem to affect charge carriers mobility. After heat treatment, the device establishes a different equilibrium state compared to that achieved at room temperature. The heat treatment worsens the P-type and improves the N-type characteristics.

\section{Acknowledgment}

The authors would acknowledge financial support from Centro Universitário da FEI, Brazil and the Brazilian funding agencies CNPq, FINEP and FAPESP. The authors would like to thank the "Centro de Tecnologia da Informação Renato Archer", Campinas, Brazil, for the sample preparation.

\section{References}

[1] S. Duzellier, Radiation effects on electronic devices in space, Aerospace Science and Technology 9 (2005), 93.

[2] J.R. Schwank, M.R. Shaneyfelt, D.M. Fleetwood, J.A. Felix, P.E. Dodd, P. Paillet, V. FerletCarvois, "Radiation Effects in MOS Oxides" IEEE Trans. on Nuc. Sci., v. 55, n. 4, p. 1883, 2008.

[3] F.F. Teixeira, C.C.M. Bordallo, M.A.G. Silveira, P.G.D. Agopian, J.A. Martino, E. Simoen and C. Claeys, "Radiation Effect on Standard and Strained Triple-Gate SOI FinFETs Parasitic Conduction", SBMicro, Curitiba, Brasil, 2013.

[4] A. Johnston, World Scientific Publishing Co. Pte. Ltd., California Inst. of Tech., USA (2010).

[5] A. S. Sedra, K. C. Smith : Microeletrônica, São Paulo. 2007

[6] W.F. Brinkman et al., IEEE Journal of Solid-State Circuits, 32, 1997.

[7] M.A.G. Silveira et al., Performance of electronic devices submitted to X-rays and high energy proton beams, Nucl. Instr. and Methods. in Physics. Research. B, v.273, p.135 - 138, 2012.

[8] V.A.P. Aguiar, et al., Experimental Setup for Single Event Effects at São Paulo 8UD Pelletron Accelerator, to appear in Nucl. Instr. and Meth. in Phys. Research B, DOI 10.1016/j.nimb.2014.02.105. 